\title{
Imaging of hypoxia in human glioblastoma with dynamic 18F-fluoromisonidazole PET
}

\author{
Redha-Alla Abdo, Frédéric Lamare², Philippe Fernandez², M'hamed Bentourkia ${ }^{1}$ \\ 'Department of Nuclear Medicine and Radiobiology, Université de Sherbrooke, Sherbrooke, QC J1H5N4, Canada. \\ ${ }^{2}$ Service de Médecine Nucléaire, Université de Bordeaux-II, EPHE, Bordeaux 33076, France.
}

Correspondence to: Dr. M'hamed Bentourkia, Department of Nuclear Medicine and Radiobiology, Université de Sherbrooke, 3001, 12th Avenue North, Sherbrooke, QC J1H5N4, Canada. E-mail: mhamed.bentourkia@usherbrooke.ca

How to cite this article: Abdo RA, Lamare F, Fernandez P, Bentourkia M. Imaging of hypoxia in human glioblastoma with dynamic 18F-fluoromisonidazole PET. Cancer Drug Resist2019;2:131-40. http://dx.doi.org/10.20517/cdr.2018.21

Received: 31 Oct 2018 First Decision: 5 Nov 2018 Revised: 11 Dec 2018 Accepted: 19 Feb 2019 Published: 19 Mar 2019

Science Editor: Godefridus J. Peters Copy Editor: Cai-Hong Wang Production Editor: Huan-Liang Wu

\begin{abstract}
Aim: The purpose of this study was to locate the levels of hypoxia in glioblastoma PET images measured with $18 \mathrm{~F}$-fluoromisonidazole in human subjects. It is recognized that tumors with hypoxia are resistant to treatment by radiotherapy and chemotherapy.
\end{abstract}

Methods: The images were acquired in dynamic mode for $15 \mathrm{~min}$ or $30 \mathrm{~min}$ and in static mode for two single scans at $2 \mathrm{~h}$ and $3 \mathrm{~h}$ to allow the accumulation of the radiotracer in the tumor. The images were analyzed at the voxel basis with compartmental analysis (CA) and with the usual tumor-to-blood uptake ratio (TBR). Kmeans algorithm was applied to cluster the levels of hypoxia in the images.

Results: TBR at a threshold of 1.2 at imaging times of $15 \mathrm{~min}, 2 \mathrm{~h}$ and $3 \mathrm{~h}$ produced images with different clusters. Also, the comparison of TBR with the distribution volume obtained with CA had a similarity index of $0.61 \pm 0.05$.

Conclusion: We found some differences in defining the hypoxic volume within a tumor using TBR. The compartmental analysis allowed discrimination of the tumor hypoxic sub-volumes which can be useful for a better treatment with radiotherapy.

Keywords: PET, 18F-fluoromisonidazole, glioblastoma, hypoxia, tumor-to-blood uptake ratio, compartmental modeling

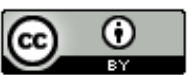

2019. Open Access This article is licensed under a Creative Commons Attribution 4.0 International ;reativecommons.org/licenses/by/4.0/), which permits unrestricted use, sharing, adaptation, distribution and reproduction in any medium or format, for any purpose, even commercially, as long as you give appropriate credit to the original author(s) and the source, provide a link to the Creative Commons license, and indicate if changes were made. 


\section{INTRODUCTION}

Glioblastoma multiforme (GBM) is known as the most common tumor in the central nervous system. The current techniques of treatment for GBM include surgery, chemotherapy and radiotherapy. These strategies of treatment have done minimum outcomes in extending the life expectancies of patients diagnosed with GBM. The patients' median survival is ranging from 1 to 1.5 years, and only $5 \%$ of the patients reach five years survival $^{[1]}$.

The difficulty of treating GBM tumors lies in its inherent histological complexity and to various mechanisms leading to treatment resistance. One of these mechanisms is hypoxia, which is defined as low oxygenation levels inside the tumor.

The apparent reason for treatment resistance due to hypoxia in chemotherapy is the tumor's distance from vasculature which prevents the therapeutic agent from reaching the tumor bed ${ }^{[2]}$. Moreover, the nonproliferation nature of hypoxic tumor can intrinsically immune the tumor to the anti-proliferative anticancer drugs ${ }^{[3]}$. The recent studies found that the molecular basis for this resistance belongs to hypoxia inducible factors, which play an essential role in tumorigenesis and angiogenesis ${ }^{[4]}$. In radiotherapy, the effect of treatment resistance due to hypoxia is associated with the oxygen effects on the radio-sensitivity of the tumor ${ }^{[5]}$. For these reasons, there are critical needs for the development of more effective therapeutic approaches.

One of the emerging strategies to overcome hypoxia is to define the hypoxic sub-volumes in the tumor using functional imaging for the goal of radiotherapy dose escalation. This hypoxia dose painting aimed to reach a biological instead of physical conformity for the delivery of radiation to the tumor. The first trial of this technique proposed by Ling et al. ${ }^{[6]}$ who applied a non-uniform radiation dose based on tumor biological characteristics.

A variety of PET tracers were used to image hypoxia, such as $18 \mathrm{~F}$-fluoromisonidazole (18F-FMISO) and $18 \mathrm{~F}$-fluoroazomycin arabinoside ${ }^{[7]}$. 18F-FMISO accumulates in the hypoxic cells for a long time $(4 \mathrm{~h})$ in vivo and in vitro. Furthermore, it requires a high level of hypoxia. However, 18F-FMISO only accumulates in viable cells with the presence of hypoxia, which can be advantageous for in vivo imaging ${ }^{[8]}$.

For hypoxia measurement in PET images, tumor-to-blood uptake ratio (TBR) is the commonly used parameter for single time frame images. It is described as the ratio of the radiotracer concentration in the tumor cells to its concentration in blood ${ }^{[9]}$. However, TBR does not give any information about the kinetics or changes of the tumor hypoxia with time. Compartmental analysis (CA) with kinetic modeling is the mostly used approach to analyze the dynamic PET data ${ }^{[10]}$. This method is based on the design of a mathematical model which best describes the physiological processes in which the tracer is involved ${ }^{[11]}$.

The objectives of this study were to demonstrate the variation of TBR with PET acquisition time, and to decompose the dynamic 18F-FMISO PET images into clusters describing hypoxia sub-volumes. This decomposition would allow the oncologists to optimize the treatment dose.

\section{METHODS}

\section{Subjects' data}

Nine human subjects with GBM tumors were imaged with PET/CT and 18F-FMISO radiotracer. 18F-FMISO was prepared using the method as described in $^{[12]}$. The PET image acquisition started with the injection of 3.7 MBq/kg of 18 F-FMISO in a dynamic acquisition of 15 min or 30 min using the PET scanner Discovery RX General Electric Medical System. Then the subjects left the scanner. Two additional static images were 
obtained for all subjects at $2 \mathrm{~h}$ and $3 \mathrm{~h}$ post-injection (pi). The images were reconstructed in 12 frames of $10 \mathrm{~s}$, 8 frames of $15 \mathrm{~s}, 11$ frames of $60 \mathrm{~s}$ and $600 \mathrm{~s}$ for the two static images. The dynamic PET images from 0 to 15 min or 30 min were coregistered with those at $2 \mathrm{~h}$ and $3 \mathrm{~h}$ static images based on the coregistration of their respective $\mathrm{CT}$ images.

\section{Data analyses}

\section{TBR analyses}

TBR images were estimated by dividing the PET images by the blood value obtained from the input function (IF) at the corresponding frame time. IF was derived for each subject from the carotid artery in PET images by drawing a region of interest (ROI) on the sagittal view to encompass a large area of the carotid. Hypoxia images were then obtained by thresholding TBR images acquired at $15 \mathrm{~min}, 2 \mathrm{~h}$ and at $3 \mathrm{~h}$, at a level of 1.2 which is the commonly used level in the literature ${ }^{[9]}$.

\section{Compartmental analysis}

We used the two-tissue compartment model for kinetic modeling of the dynamic images ${ }^{[13,14]}$ at the voxel basis in order to obtain parametric images. This model is based on the physiological properties of $18 \mathrm{~F}-\mathrm{FMSIO}$ representing the exchange of the radiotracer with blood stream in the perfused compartment of the tumor and its accumulation in the hypoxic compartment.

The time-activity curve (TAC) of the radiotracer concentration in blood was assessed by drawing an ROI on the carotid artery from summed sagittal images on the first 4 min where the images show only blood. The large sagittal images of the carotids allowed to minimize the effect of partial volume. The ROIs on the tumors were fitted with the two-tissue compartment model ${ }^{[13,14]}$ using the Levenberg-Marquardt algorithm. The adjustment of the tumor TAC in each pixel returned the four rate constants $K_{1}-k_{4}$ and blood volume.

The distribution volume was estimated as $K_{1} /\left(k_{2}+k_{3}\right)^{[13]}$, where $K_{1}$ is the perfusion rate constant, $k_{2}$ is the brain to blood transport rate constant (reverse capillary membrane transport), and $k_{3}$ is the $18 \mathrm{~F}-\mathrm{FMISO}$ retention rate constant in the tumor. Distribution volume is a more sensitive parameter for hypoxia determination as it depends on the perfusion $K_{1}$ and on the extraction and elimination rate constants $k_{3}$ and $k_{2}$ respectively ${ }^{[13,14]}$, instead of relying on the perfusion rate constant $K_{1}$ as reported in $^{[15]}$ where the authors compared $K_{1}$ to the perfusion measured with perfusion CT imaging in lung cancer.

\section{Statistical analyses}

CA modeling and statistical analyses were implemented in MATLAB (R2015a, The Math Works Inc., Natick, MA, USA). The CA distribution volume was analyzed using Kmeans and elbow method ${ }^{[16]}$. The comparison between TBR and CA images was assessed using the similarity index $(\mathrm{SI})^{[17]}$ :

$$
\operatorname{SI}(x, y)=\left[\left(2 \mu_{x} \mu_{y}\right)\left(2 \sigma_{x y}\right)\right] /\left[\left(\mu_{x}^{2}+\mu_{y}^{2}\right)\left(\sigma_{x}^{2}+\sigma_{y}^{2}\right)\right]
$$

Where $\mu_{x}, \mu_{y}, \sigma_{x}, \sigma_{y}$ and $\sigma_{x y}$ are the local means, standard deviations, and cross-covariance for images $x$ and $y$. The SI value of 1 indicates a complete agreement while 0 indicates a disagreement between the two images.

The elbow method is utilized to select the optimal number of clusters in a dataset as returned by Kmeans. For each number of clusters $k$, say from 1 to 10 , the sum of the squared errors are calculated and plotted as a function of the values of $k$, which can be seen to sharply drop at a certain value of $k$. This drop indicates the maximum of clusters needed ${ }^{[18]}$. 
A

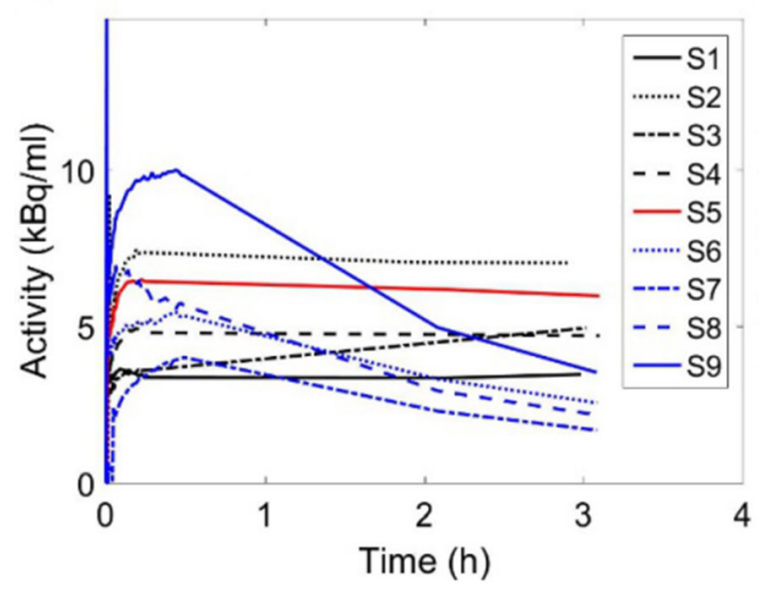

B

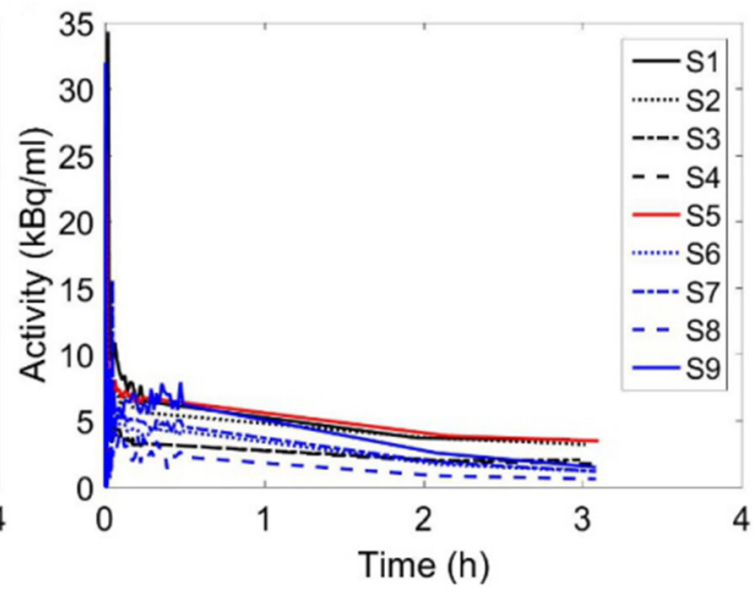

Figure 1. A: Tumor time activity curves in the nine subjects; $B$ : their corresponding input functions

\section{RESULTS}

Tumor TACs defined by drawing ROIs on the tumor images from the nine subjects are shown in Figure 1A. The uptake of $18 \mathrm{~F}-\mathrm{FMISO}$ presented different behavior as a function of time among subjects. The increasing TACs were related to hypoxic tumors accumulating $18 \mathrm{~F}-\mathrm{FMISO}$, those having a plateau represented stable and uniform hypoxia including necrotic sub-volumes, and those with decreasing TACs were related to perfused tumors with less hypoxia. Figure $1 \mathrm{~B}$ shows the IFs in the respective subjects as for the TACs.

Figure 2 first column depicts images of the nine subjects taken from the image frames at $3 \mathrm{~h}$, where the tumor ROIs were also drawn. ROIs in some subjects are shown to encompass deeply contrasted volumes (Figure 2, first column: S3, S4, S6 and S7) which we assumed to be necrotic sub-volumes. In all images, at $3 \mathrm{~h}$, the tumor appeared well contrasted from the rest of the brain which was perfused, indicating that the exchange of $18 \mathrm{~F}-\mathrm{FMISO}$ between the tumor and blood is slow in comparison to the exchange between normal tissue and blood (Figure 2, first column).

Figure 2 shows also TBR for $15 \mathrm{~min}, 2 \mathrm{~h}$ and $3 \mathrm{~h}$ pi after 1.2 thresholding. TBR translated the contrast between the tumor and the blood, and consequently between hypoxic and perfused tissues. Depending on the time of the PET measurement, the ratio of tissue to blood radiotracer concentration can drastically vary. At $15 \mathrm{~min}$, the radiotracer concentration in blood is high while the uptake in the tumor has not been optimally achieved (see Figure 1A for tissue and Figure 1B for blood), and consequently TBR values partly isolate tumor hypoxia as shown in Figure 2 second column. For this reason, it has been proposed to delay the PET imaging at $2 \mathrm{~h}$ pi or later in order to allow more uptake of the radiotracer in the tumor and concurrently its washout from blood. Still TBR at $2 \mathrm{~h}$ and $3 \mathrm{~h}$ shows differences in tumor hypoxia definition (Figure 2, columns 3 and 4). Therefore, the time for a single PET scan to isolate hypoxia remains questionable. The large perfused regions in the images defined with TBR can also be explained by the underestimation of the tracer activity in blood as determined from the carotid artery, and this might be partly due to the partial volume effect.

The TBR images at $15 \mathrm{~min}, 2 \mathrm{~h}$ and $3 \mathrm{~h}$ highlighted the difference in tumor volume determination which was varying with time. Such assumptions can lead to different results depending on the protocol for 18F-FMISO imaging. The last column in Figure 2 represents the CA distribution volume for each subject. These images can help in understanding the kinetics of $18 \mathrm{~F}$-FMISO with time. The similarity test between TBR images at $3 \mathrm{~h}$ and the corresponding CA distribution volume images was mean $(\mathrm{SI})=0.61 \pm 0.05$. 


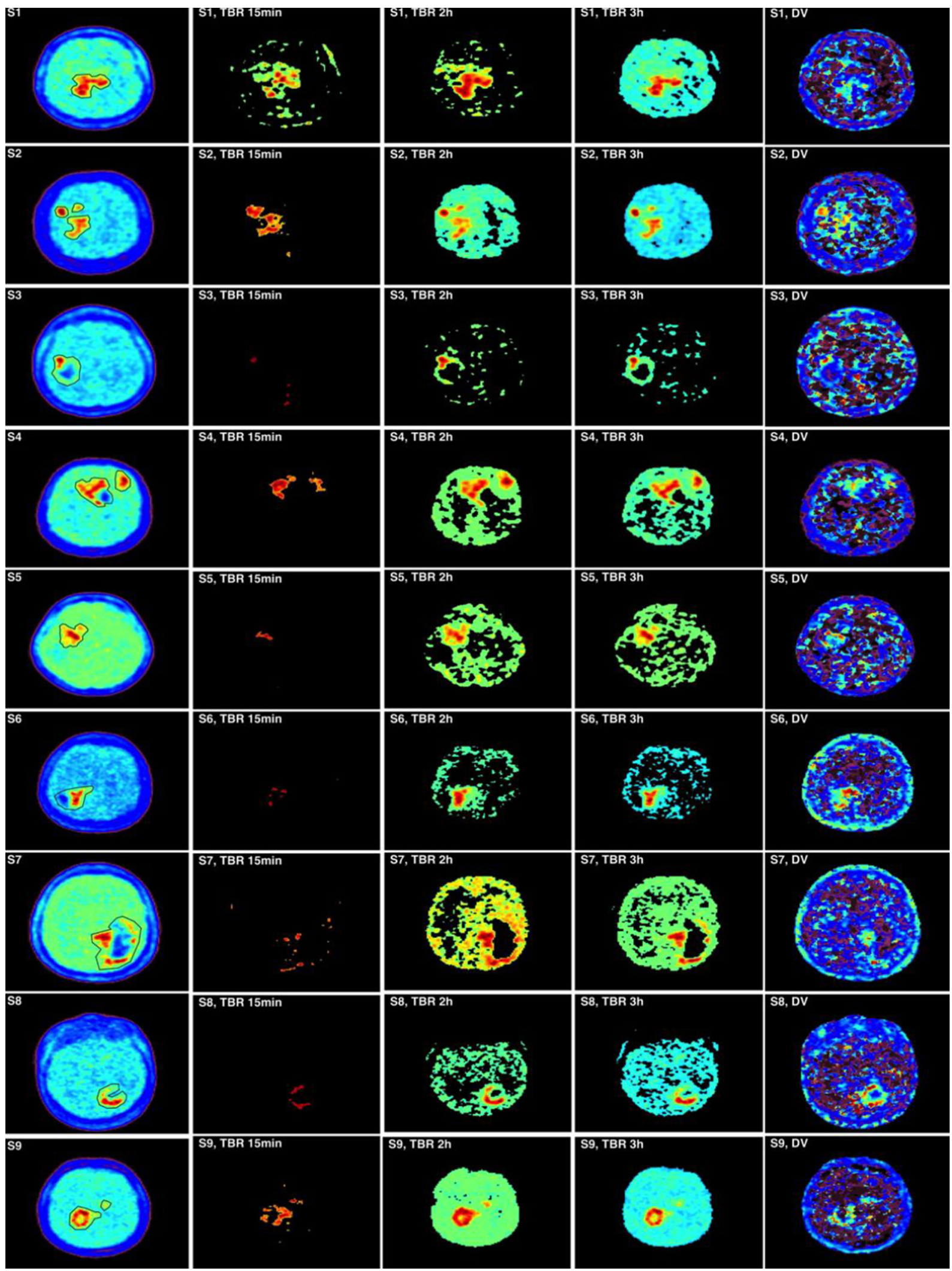

Figure 2. Comparison of tumor-to-blood uptake ratio (TBR) at $15 \mathrm{~min}, 2 \mathrm{~h}$ and $3 \mathrm{~h}$ with compartmental analysis (CA) images. The first column shows the measured PET images at $3 \mathrm{~h}$ pi with region of interests around the tumor. The three-centered columns are for the thresholded TBR images at a threshold of 1.2. The fifth column is the corresponding CA distribution volume 
The clustering to discriminate the levels of hypoxia from the distribution volume images are shown in Figure 3 for three subjects. Based on the determination of the clusters, the TACs were produced to show the time course for each cluster [Figure 3]. The three subjects' data in Figure 3 were selected to show the accumulation with time of $18 \mathrm{~F}-\mathrm{FMISO}$ as increasing, stabilizing and decreasing. Again, CA images were shown to provide different structures of the tumor depending on its uptake of 18F-FMISO. The clusters presented different time course shapes and sub-volumes within the tumor in each subject and these might have reflected the levels of hypoxia.

Figure 4 was obtained from the cluster TACs in Figure 3 divided at time points by IF (Figure 4 left column) and by a contralateral normal tissue TAC (Figure 4 right column). In these plots, a horizontal straight line has been added at the level 1.2 to indicate the threshold used in TBR to separate hypoxic from non-hypoxic clusters. Clustering hypoxia reflects more its heterogeneity and possibly provides more accurate treatments.

Figure 5 depicts the behavior of the cluster TACs in the same image slice by including or excluding the necrotic region in the tumor. A thresholding or a clustering can resolve the depressed regions in the tumor after application of CA.

\section{DISCUSSION}

There are many major challenges in defining the hypoxic region with 18F-FMISO PET images. For example, the accumulated activity in the voxel depends on the volume of hypoxia in the tumor. Furthermore, the transport properties and acquisition time affect the uptake of 18F-FMISO. Defining the exact volume of hypoxia within GBM tumors can help to overcome the treatment resistance in chemo-radiotherapy. Most PET imaging in radiotherapy used single static scan at $2 \mathrm{~h}$ to $4 \mathrm{~h}$ pi to allow for 18F-FMISO accumulation in the target cells.

The current assessment of hypoxia using TBR or standard uptake value shows the uptake at a specific time point. Therefore, their results would change by different time measurements ${ }^{[\rho]}$. This observation is clearly indicated in Figures 1 and 2 where the tumor hypoxic volumes were different between the $15 \mathrm{~min}, 2 \mathrm{~h}$ and $3 \mathrm{~h}$. By using TBR, the boost dose of radiation treatment to the hypoxic region would depend on the scanning time of $18 \mathrm{~F}-\mathrm{FMISO}$. In addition to scanning time, the threshold value of TBR has not yet been well established. We used the threshold value of 1.2 in this study, while other studies suggesting the value of 1.4. In the literature, many threshold values have been used for delineating the hypoxic volume other than 1.2 or 1.4. In addition, the uncertainties on the $18 \mathrm{~F}-\mathrm{FMISO}$ activity in blood have a reasonable impact on hypoxia determination.

By using CA technique, it was possible to differentiate the voxels inside tumor hypoxic volume into different levels of hypoxia. These differences resulted from the fact that TBR would use only a single time data, while CA evaluates the data using the whole dynamic images. The patterns of the cluster TACs showed different behavior other than the previous shape of the whole tumor (Figure $3 \mathrm{vs}$. Figure 1). Thorwarth et al. ${ }^{[19]}$ had described four examples of TACs with 18F-FMISO. These were the well-perfused region TAC, diffusionlimited hypoxia, diffusion-limited with structural hypoxia and high hypoxic/necrotic area.

About hypoxia sub-volumes and levels, most of authors defined two tumor sub-volumes as volumes with signal intensity below or above a given threshold usually taken as $\mathrm{TBR}=1.2$. Because hypoxia is spatially heterogeneous, the thresholding normally generates clusters of voxels with TBR $>1.2$. The same pattern is obtained when applying compartmental modeling ${ }^{[19]}$. Moreover, a single voxel can be made of perfused and hypoxic sub-volumes ${ }^{[19]}$. Since clusters of hypoxic sub-volumes can be found at different locations in the tumor, these clusters can have in average different signals (or $\mathrm{Bq} / \mathrm{mL}$ ) which can be referred to as hypoxia 

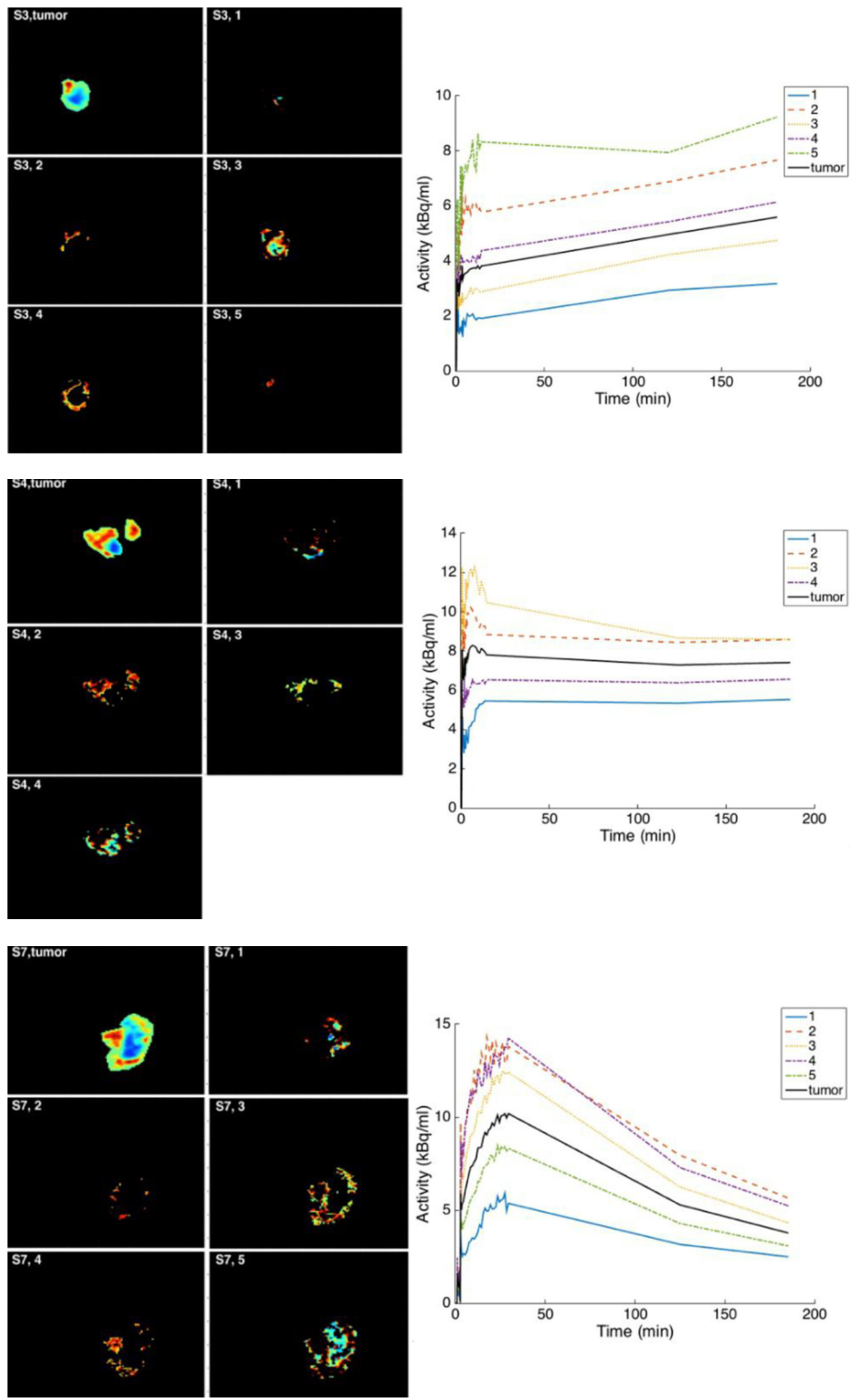

Figure 3. The separation of compartmental analysis (CA) distribution volume based on Kmeans clustering and elbow method. From upper to bottom: S3, S4 and S7. The displayed image of the whole tumor is from the measured image. For each cluster determined on CA distribution volume, its time-activity curve was extracted from the measured images. In these subjects, the accumulation of $18 \mathrm{~F}$-fluoromisonidazole is seen to respectively increase, stabilize and decrease 

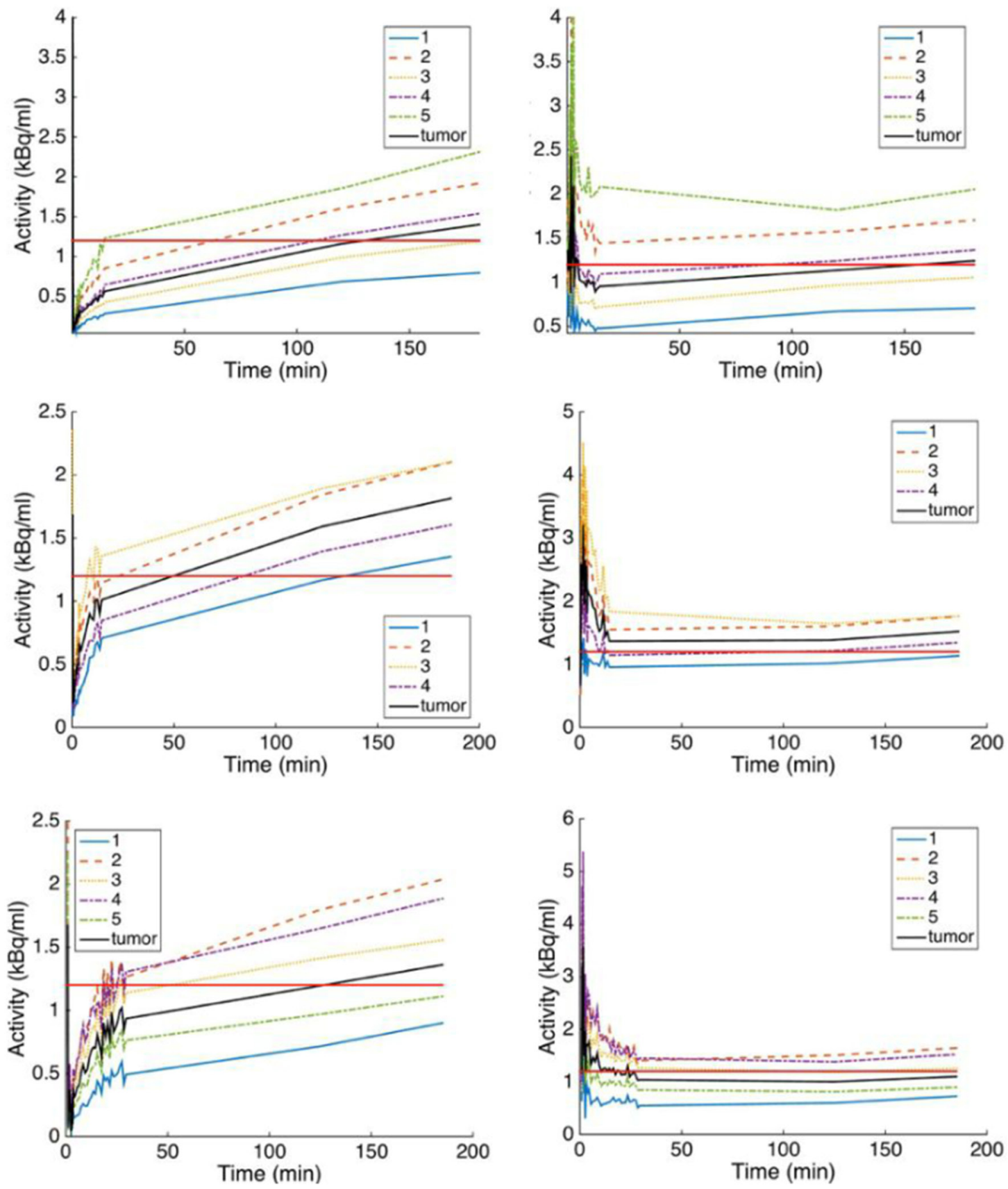

Figure 4. The time-activity curves (TACs) of the distribution volume clusters in Figure 3 were normalized here with IF (left column) and with TAC obtained from a contralateral normal tissue. The straight line represents the threshold of 1.2 above which the cluster was considered as hypoxic

levels. Dirix et al. ${ }^{[20]}$ and Zips et al. ${ }^{[21]}$ reported the correlation of radiation treatment with hypoxia magnitude as assessed with $\mathrm{T} / \mathrm{Bmax}^{[20]}$ and with $\mathrm{TBR}=1.4,1.6,1.8$ and $2^{[21]}$.

In conclusions, we showed in this work some differences in defining the hypoxic volumes within a tumor using TBR with the single static imaging protocol, while the compartmental analysis allowed to determine the levels of hypoxia within the tumor sub-volumes. Because GBM behaves differently from one subject to another, it is suggested to apply the appropriate analysis approach instead of using a common method like TBR. The 30 min dynamic PET data could be appropriate and sufficient to isolate hypoxia levels. Although 

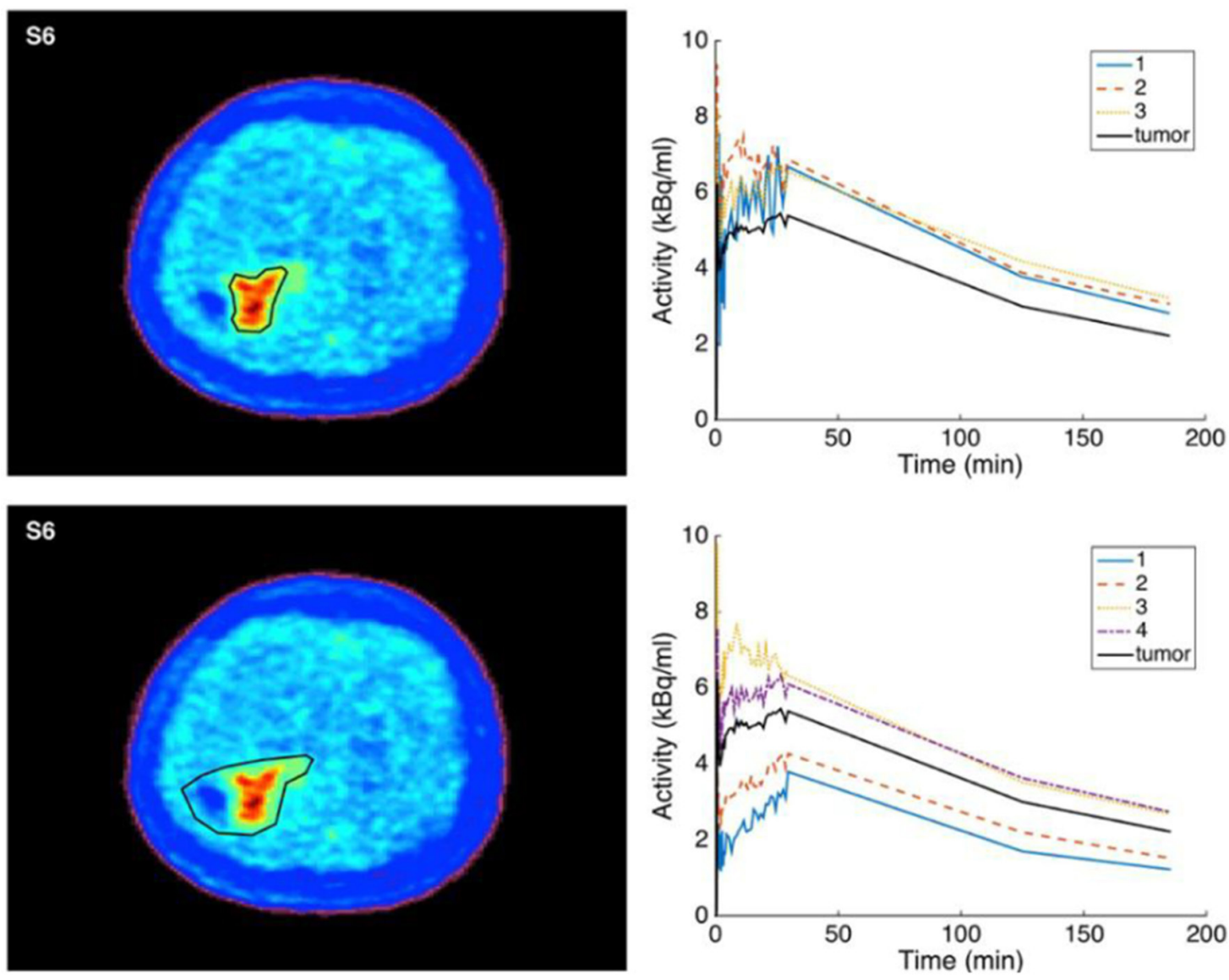

Figure 5. The effect of clustering when including or excluding tumor depressed regions (after surgery without presence of inflammation or in presence of necrosis)

this approach seems to have potential, more validations are required for its appropriate application. This might possibly be achieved in a randomized study.

\section{DECLARATIONS}

\section{Authors' contributions}

Data analysis, writing the original draft: Abdo RA

Data collection, resources, revision: Lamare F, Fernandez P

Conceptualization, methodology, software, revision of the original draft: Bentourkia M

\section{Data source and availability}

Corresponding author may be contacted for any data inquiries.

\section{Financial support and sponsorship}

None.

\section{Conflicts of interest}

All authors declared that there are no conflicts of interest.

\section{Ethical approval and consent to participate}

This research study was approved by the institutional review board of the study site. 


\section{Consent for publication}

Not applicable.

\section{Copyright}

(c) The Author(s) 2019.

\section{REFERENCES}

1. Gilbert MR. Recurrent glioblastoma: a fresh look at current therapies and emerging novel approaches. Semin Oncol 2011;38:S21-33.

2. Vaupel P, Kelleher DK, Höckel M. Oxygen status of malignant tumors: pathogenesis of hypoxia and significance for tumor therapy. Semin Oncol 2001;28:29-35.

3. Oliver L, Olivier C, Marhuenda F, Campone M, Vallette F. Hypoxia and the malignant glioma microenvironment: regulation and implications for therapy. Curr Mol Pharmacol 2009;2:263-84.

4. Comerford KM, Wallace TJ, Karhausen J, Louis NA, Montalto MC, et al. Hypoxia-inducible factor-1-dependent regulation of the multidrug resistance MDR1 gene. Cancer Res 2002;62:3387-94.

5. Weeks AJ, Paul RL, Marsden PK, Blower PJ, Lloyd DR. Radiobiological effects of hypoxia-dependent uptake of $64 \mathrm{Cu}-\mathrm{ATSM}$ : enhanced DNA damage and cytotoxicity in hypoxic cells. Eur J Nucl Med Mol Imaging 2010;37:330-8.

6. Ling CC, Humm J, Larson S, Amols H, Fuks Z, et al. Towards multidimensional radiotherapy (MD-CRT): biological imaging and biological conformality. Int J Radiat Oncol Biol Phys 2000;47:551-60.

7. Hirata K, Terasaka S, Shiga T, Hattori N, Magota K, et al. 18F-fluoromisonidazole positron emission tomography may differentiate glioblastoma multiforme from less malignant gliomas. Eur J Nucl Med Mol Imaging 2012;39:760-70.

8. Miyake K, Ogawa D, Okada M, Hatakeyama T, Tamiya T. Usefulness of positron emission tomographic studies for gliomas. Neurol Med Chir (Tokyo) 2016;56:396-408.

9. Fleming IN, Manavaki R, Blower PJ, West C, Williams KJ, et al. Imaging tumour hypoxia with positron emission tomography. Br J Cancer 2015;112:238-50.

10. Sokoloff L, Reivich M, Kennedy C, Des Rosiers MH, Patlak CS, et al. The [14C] deoxyglucose method for the measurement of local cerebral glucose utilization: theory, procedure, and normal values in the conscious and anesthetized albino rat. J Neurochem 1977;28:897-916.

11. Li F, Joergensen JT, Hansen AE, Kjaer A. Kinetic modeling in PET imaging of hypoxia. Am J Nucl Med Mol Imaging. 2014;4:490-506.

12. Lim JL, Berridge MS. An efficient radiosynthesis of [18F]fluoromisonidazole. Appl Radiat Isot 1993;44:1085-91.

13. Phelps ME, Huang SC, Hoffman EJ, Selin C, Sokoloff L, et al. Tomographic measurement of local cerebral glucose metabolic rate in humans with (F-18)2-fluoro-2-deoxy-D-glucose: validation of method. Ann Neurol 1979;6:371-88.

14. Bruehlmeier M, Roelcke U, Schubiger PA, Ametamey SM. Assessment of hypoxia and perfusion in human brain tumors using PET with 18F-fluoromisonidazole and 15O-H2O. J Nucl Med 2004;45:1851-9.

15. McGowan DR, Skwarski M, Papiez BW, Macpherson RE, Gleeson FV, et al. Whole tumor kinetics analysis of 18F-fluoromisonidazole dynamic PET scans of non-small cell lung cancer patients, and correlations with perfusion CT blood flow. EJNMMI Res 2018;8:73.

16. Bholowalia P, Kumar A. Ebk-means: a clustering technique based on elbow method and K-Means in WSN. Int J Comput Appl Vol $2014 ; 105$.

17. Wang Z, Bovik AC, Sheikh HR, Simoncelli EP. Image quality assessment: From error visibility to structural similarity. IEEE Trans Image Process 2004;13:600-12.

18. Syakur MA, Khotimah BK, Rochman EMS, Satoto BD. Integration K-means clustering method and elbow method for identification of the best customer profile cluster. IOP Conference Series: Materials Science and Engineering 2018; doi: 10.1088/1757$899 \mathrm{X} / 336 / 1 / 012017$.

19. Thorwarth D, Eschmann SM, Paulsen F, Alber M. A kinetic model for dynamic [18F]-Fmiso PET data to analyse tumour hypoxia. Phys Med Biol 2005;50:2209-24.

20. Dirix P, Vandecaveye V, De Keyzer F, Stroobants S, Hermans R, et al. Dose painting in radiotherapy for head and neck squamous cell carcinoma: value of repeated functional imaging with 18F-FDG PET, 18F-fluoromisonidazole PET, diffusion-weighted MRI, and dynamic contrast-enhanced MRI. J Nucl Med 2009;50:1020-7.

21. Zips D, Zöphel K, Abolmaali N, Perrin R, Abramyuk A, et al. Exploratory prospective trial of hypoxia-specific PET imaging during radiochemotherapy in patients with locally advanced head-and-neck cancer. Radiother Oncol 2012;105:21-8. 